\title{
Dynamical treatment of fission fragment angular distribution
}

\author{
A V Karpov ${ }^{1}$, R M Hiryanov ${ }^{2}$, A V Sagdeev ${ }^{2}$ and G D Adeev ${ }^{2}$ \\ ${ }^{1}$ Flerov Laboratory of Nuclear Reactions, JINR, Dubna, 141980, Russia \\ 2 Department of Theoretical Physics, Omsk State University, Omsk, 644077, Russia \\ E-mail: karpov@jinr.ru,hirjanov@mail.ru and adeev@omsu.ru
}

Received 25 July 2006

Published 11 December 2006

Online at stacks.iop.org/JPhysG/34/255

\begin{abstract}
A dynamical approach to the treatment of fission fragment angular distribution is developed. The approach is based on three-dimensional Langevin dynamics for shape collective coordinates joined with the Monte Carlo algorithm for the degree of freedom associated with the projection $K$ of the total angular momentum of the fissioning system on the symmetry axis. The relaxation time of the tilting mode $\tau_{K}$ is estimated. From fits to experimental data on the fission fragment angular distribution of heavy fissioning compound systems, the $K$ equilibration time is deduced to be $\sim 4 \times 10^{-21}$ s for temperatures $\sim 1-2 \mathrm{MeV}$. A modified one-body mechanism of nuclear viscosity with the reduction coefficient of the contribution from the 'wall' formula $k_{s}=0.25$ has been used in calculations.
\end{abstract}

\section{Introduction}

The transition-state model is conventionally used in theoretical analysis of the data on the angular distribution of fission fragments [1-3]. The essence of this model consists of the assumption that there is a certain chosen (transition) configuration of a fissioning system that determines the angular distribution of fission fragments. Thus, there are two limiting assumptions on the position of the transition state and, correspondingly, two variants of the transition-state model: the saddle-point transition-state (SPTS) model [1-3] and the scission-point transition-state (SCTS) model [4-6]. The SPTS model gives a reasonably exact reproduction of the experimental data on the anisotropy of the fission fragment angular distributions for reactions in which neutrons, ${ }^{3} \mathrm{He}$ ions, and $\alpha$ particles were used as projectiles $[1,7]$. The compound nuclei formed in such reactions have a temperature of about $1 \mathrm{MeV}$ and low angular momenta.

It was found that the standard SPTS model regularly predicted low values of the angular distribution anisotropy for reactions with more massive ions of carbon, oxygen and heavier 
ions [7], and the experimental data are closer to the values obtained according to the SCTS model.

Considerable success in describing the angular distribution in reactions with heavy ions was achieved with the version of the SCTS model developed in [6]. The authors of this study proposed a model which more accurately (in comparison with the first variants of the SCTS model $[4,5]$ ) took into account the spin (twisting and wriggling) modes of formed fission fragments.

At the same time, it was shown in [8] that the experimentally observed angular distribution anisotropy cannot be described by either the SPTS or SCTS models. Therefore, it was assumed, in the general case, that the transition state determining the angular distribution of fission fragments is located somewhere between the saddle point and the scission point.

The most general solution of the angular distribution problem can be achieved by taking into account the dynamical features of the formation of the angular distribution. In the present paper, a dynamical approach to the treatment of the fission fragment angular distribution is developed. The approach is based on the combining of three-dimensional Langevin dynamics for collective shape coordinates with the stochastic dynamics of the tilting mode of fission. The results of dynamical calculations of the fission fragment angular distributions from the compound systems formed in fusion-fission reactions with heavy ions are presented. The calculations were carried out within a four-dimensional model based on the Langevin equations for the reactions: ${ }^{16} \mathrm{O}+{ }^{232} \mathrm{Th} \rightarrow{ }^{248} \mathrm{Cf},{ }^{16} \mathrm{O}+{ }^{238} \mathrm{U} \rightarrow{ }^{254} \mathrm{Fm},{ }^{16} \mathrm{O}+{ }^{248} \mathrm{Cm} \rightarrow{ }^{264} \mathrm{Rf}$. The main quantity that determines the dynamics of the angular distribution formation, namely the relaxation time of the tilting mode (the mode associated with the projection of the total angular momentum on the nuclear symmetry axis), has been extracted from an analysis of the experimental data. The paper is organized as follows. The multidimensional Langevin model for the description of the dynamics of collective shape coordinates and the Monte Carlo algorithm employed for modelling the evolution of the tilting mode are described in section 2 . We recall the main features of the used Langevin model in order to make the present paper self-contained, although one can find an extended review of the multidimensional Langevin model in our previous publications [9-13] including the review [12]. The results obtained in this study and their discussion are presented in section 3. Finally, concluding remarks and a summary are given in section 4 .

\section{Model}

\subsection{Multidimensional Langevin equations}

We have presented and discussed in detail the three-dimensional Langevin model with various input parameters in our previous publications [9-13]. In the present paper, we will use the same notation and ingredients constituting the model briefly described below. In our dynamical calculations, we use the well-known 'funny hills' $\{c, h, \alpha\}$ parametrization [14]. Here, $c$ is the elongation parameter. The length of the nucleus is equal to $2 c$ in units of the spherical nucleus radius $R_{0}$. The parameter $h$ describes a variation in the thickness of the neck for a given elongation of the nucleus. The value $h=0$ corresponds approximately to the bottom of the liquid drop model [14]. The parameter $\alpha$ of mass asymmetry (mirror asymmetry) determines the ratio of the volumes (masses) of the nascent fragments. In the case $\alpha=0$ symmetric shapes are obtained, ranging from a spherical shape $(c=1, h=0)$ to two fragments shapes. For the case $\alpha \neq 0$ different asymmetric shapes are obtained. In [9], we have introduced a new mass-asymmetry parameter scaled with the elongation $\alpha^{\prime}=\alpha c^{3}$ which retains the same 
meaning as $\alpha$. Thus, we use $\left(c, h, \alpha^{\prime}\right)$ as collective coordinates in our modelling of the fission process.

The multidimensional Langevin equations are given in [15] in the difference form convenient for numerical simulation as

$$
\begin{aligned}
& p_{i}^{(n+1)}=p_{i}^{(n)}-\left[\frac{1}{2} p_{j}^{(n)} p_{k}^{(n)}\left(\frac{\partial \mu_{j k}(\mathbf{q})}{\partial q_{i}}\right)^{(n)}+\frac{\partial F^{(n)}(\mathbf{q})}{\partial q_{i}}+\gamma_{i j}^{(n)}(\mathbf{q}) \mu_{j k}^{(n)}(\mathbf{q}) p_{k}^{(n)}\right] \tau+\theta_{i j}^{(n)} \xi_{j}^{(n)} \sqrt{\tau}, \\
& q_{i}^{(n+1)}=q_{i}^{(n)}+\frac{1}{2} \mu_{i j}^{(n)}(\mathbf{q})\left(p_{j}^{(n)}+p_{j}^{(n+1)}\right) \tau,
\end{aligned}
$$

where $\mathbf{q}=\left(c, h, \alpha^{\prime}\right)$ are the collective coordinates, $\mathbf{p}=\left(p_{c}, p_{h}, p_{\alpha^{\prime}}\right)$ are the conjugate momenta, $F(\mathbf{q})=V(\mathbf{q})-a(\mathbf{q}) T^{2}$ is the Helmholtz free energy, $V(\mathbf{q})$ is the zero-temperature potential energy, $m_{i j}\left(\left\|\mu_{i j}\right\|=\left\|m_{i j}\right\|^{-1}\right)$ is the tensor of inertia, $\gamma_{i j}$ is the friction tensor. The normalized random variable $\xi_{i}$ is assumed to be white noise. The strength of the random force $\theta_{i j}$ is given by $\theta_{i k} \theta_{k j}=T \gamma_{i j}$. The temperature of the heat bath $T$ has been determined by the Fermi-gas formula $T=\left(E_{\text {int }} / a(\mathbf{q})\right)^{1 / 2}$, where $E_{\text {int }}$ is the internal excitation energy of the nucleus and $a(\mathbf{q})$ is the level-density parameter, which has been taken from the work of Ignatyuk et al [16]. It should be noted that the asymptotic level-density parameter obtained by Ignatyuk and his co-workers is in surprisingly good agreement with the level-density parameter, calculated [17] in a consistent way by using a temperature-dependent finite-range liquid drop model [18]. The repeated indices in the equation above imply summation over the collective coordinates from 1 to 3 .

The upper index in equation (1) denotes that the related quantity is calculated on the $n$th time step. The internal excitation energy is determined by using the energy conservation law:

$$
E^{*}=E_{\mathrm{int}}+E_{\mathrm{coll}}(\mathbf{q}, \mathbf{p})+V(\mathbf{q})+E_{\mathrm{evap}}(t),
$$

where $E^{*}$ is the total excitation energy of the nucleus, $E_{\text {coll }}(\mathbf{q}, \mathbf{p})=\frac{1}{2} \mu_{i j}(\mathbf{q}) p_{i} p_{j}$ is the kinetic energy of the collective degrees of freedom and $E_{\text {evap }}(t)$ is the energy carried away by evaporated particles by the time $t$. The potential deformation energy $V(\mathbf{q})$ in the finite-range liquid drop model based on the Yukawa-plus-exponential mass formula is a function of the mass number $A=N+Z$, relative neutron excess $(N-Z) / A$, total angular momentum $I$, its projection $K$ on the symmetry axis and a set of collective coordinates $\mathbf{q}$. The following expression has been suggested in [18-20] for $V(\mathbf{q})$ :

$$
\begin{aligned}
V(A, Z, \mathbf{q}, I, K) & =a_{\mathrm{s}} A^{2 / 3}\left(1-k\left(\frac{N-Z}{A}\right)^{2}\right)\left[B_{\mathrm{n}}(\mathbf{q})-B_{\mathrm{n}}\left(\mathbf{q}_{\mathrm{gs}}\right)\right] \\
+ & a_{\mathrm{c}} \frac{Z^{2}}{A^{2 / 3}}\left[B_{\mathrm{c}}(\mathbf{q})-B_{\mathrm{c}}\left(\mathbf{q}_{\mathrm{gs}}\right)\right]+\frac{\hbar^{2}\left(I(I+1)-K^{2}\right)}{2 J_{\perp}(\mathbf{q})}+\frac{\hbar^{2} K^{2}}{2 J_{\|}(\mathbf{q})},
\end{aligned}
$$

where $a_{\mathrm{s}}, a_{\mathrm{c}}$ are the usual surface and Coulomb energy parameters of the finite-range liquid drop model at zero temperature and $k$ is the surface coefficient of the symmetry energy [20]. The deformation dependences are taken into account through the shape functionals $B_{\mathrm{n}}(\mathbf{q}), B_{\mathrm{c}}(\mathbf{q}), J_{\perp}(\mathbf{q})$ and $J_{\|}(\mathbf{q})$. The functionals of generalized nuclear energy $B_{\mathrm{n}}(\mathbf{q})$ and Coulomb energy $B_{\mathrm{c}}(\mathbf{q})$ model the effects of the finite range of nuclear forces and the realistic distributions of charge and nuclear densities. These functionals in the finite-range liquid drop model are no longer independent of the size of the nuclear drop and must be calculated for any specific nucleus in contrast to the liquid drop model with a sharp surface. For spherical nuclei they are also no longer equal to 1, but they can be evaluated in closed form [19].

The last two terms in equation (3) represent the rotational energy depending on the total momentum of compound nucleus $I$ and its projection on the nucleus symmetry axis $K$. The functionals $J_{\perp}(\mathbf{q})$ and $J_{\|}(\mathbf{q})$ are the rigid-body moments of inertia at deformation $\mathbf{q}$ with 
respect to the axis perpendicular to the symmetry axis and to the symmetry axis, respectively. According to [21] these functionals can be calculated taking into account the diffuseness of realistic nuclear density distribution in the following way:

$$
J_{\perp(\|)}(\mathbf{q})=J_{\perp(\|)}^{(\text {sharp })}+4 M a_{M}^{2},
$$

where $J_{\perp(\|)}^{\text {(sharp) }}$ are the moments of inertia obtained within the sharp-surface liquid drop model, $a_{M}=0.704 \mathrm{fm}$ is the diffuseness parameter of the nuclear matter distribution and $M$ is the mass of the compound nucleus.

A modified one-body mechanism of nuclear viscosity $[22,23]$ has been used in order to determine the dissipative part of the driving force. The expressions applied to calculate the friction tensor within the so-called surface-plus-window dissipation model can be found in our previous works $[9,12]$. We used the reduction coefficient value $k_{s}=0.25$. The coefficient $k_{s}$ determines the strength of nuclear dissipation. This value is very close to that extracted from experimental analysis [22] of the widths of giant resonances $\left(k_{s}=0.27\right)$. Also, our previous calculations $[9,10]$ have shown that the experimental data on the mean prescission neutron multiplicity and variance of the mass-energy distribution can be reproduced with the $k_{s}$ coefficient in the range $k_{s}=0.25-0.5$. The inertia tensor is calculated by means of the Werner-Wheeler approximation.

Langevin trajectories are simulated starting from the ground state with the excitation energy $E^{*}$ of the compound nucleus. The initial conditions were chosen by the Neumann method with generating function

$$
P\left(\mathbf{q}_{0}, \mathbf{p}_{0}, l_{0}, t=0\right) \sim \exp \left[-\frac{V\left(\mathbf{q}_{0}\right)+E_{\mathrm{coll}}\left(\mathbf{q}_{0}, \mathbf{p}_{0}\right)}{T}\right] \delta\left(\mathbf{q}_{0}-\mathbf{q}_{\mathrm{gs}}\right) \frac{\mathrm{d} \sigma(l)}{\mathrm{d} l} .
$$

Thus, we start modelling the fission dynamics from the spherical nucleus, i.e. $\mathbf{q}_{\mathrm{gs}}=(c=1$, $\left.h=0, \alpha^{\prime}=0\right)$. The initial state is assumed to be characterized by thermal equilibrium momentum distribution and by spin distribution $\mathrm{d} \sigma(l) / \mathrm{d} l$ for the complete fusion of heavy ions. We have parametrized the spin distribution of compound nuclei $\mathrm{d} \sigma(l) / \mathrm{d} l$ according to scaled prescription [15], which reproduces to a certain extent the dynamical results of the surface friction model [24] for the fusion of two heavy ions.

It has been supposed that the division of a mononucleus into fragments occurred when the neck radius of the fissioning nucleus is equal to $0.3 R_{0}[14,25]\left(R_{0}\right.$ is the radius of the initial spherical nucleus). This scission condition determines the scission surface in the space of collective coordinates. The scission configuration is determined by the intersection points of a stochastic Langevin trajectory of the fissioning system with the scission surface in the coordinate subspace.

The evaporation of prescission light particles $(j=n, p, \alpha, \gamma)$ along Langevin trajectories was taken into account using a Monte Carlo simulation technique $[15,26]$. All the dimensional factors were recalculated when a light prescission particle was evaporated, only the dimensionless functionals of the rotational, Coulomb and nuclear energies were not recalculated. The loss of the angular momentum was taken into account by assuming that light particles carry away $l_{j}=1,1,2,1(\hbar)$ [15]. These computations are obligatory, because the evaporation of light particles results in the change of the nucleonic composition and loss of the angular momentum of the initial compound nucleus.

\subsection{Formalism of calculation of fission fragment angular distribution}

In the analysis of the angular distributions, it is usually assumed that fission fragments are emitted in the direction of the nuclear symmetry axis. In this case, the angular distribution is 
specified by three quantum numbers: $I, K$ and $M$, where $I$ is the total angular momentum of a compound nucleus, $K$ and $M$ are the projections of $I$ on, respectively, the nuclear symmetry axis and the beam direction.

In the case of the fusion of spinless ions, the value of $M=0$. For this case, the partial fusion cross section $\mathrm{d} \sigma(l) / \mathrm{d} l$ becomes the distribution of compound nuclei with respect to the total angular momentum $\sigma_{I}$. Then, the angular distribution for the fixed values of $I$ and $K$ has the form [1]

$$
W(\theta, I, K)=(I+1 / 2)\left|d_{M=0, K}^{I}(\theta)\right|^{2},
$$

where $d_{M=0, K}^{I}(\theta)$ is the Wigner rotation function (the explicit expression for $d_{M, K}^{I}(\theta)$ can be found in monograph [1]), $\theta$ is the angle between the nuclear symmetry axis and the beam axis.

The angular distribution of fission fragments observed in experiment can be obtained by averaging equation (6) over the quantum numbers $I$ and $K$ :

$$
W(\theta)=\sum_{I=0}^{\infty} \sigma_{I} \sum_{K=-I}^{I} P(K) W(\theta, I, K) .
$$

It is seen from equation (7) that for the calculation of the angular distribution it is necessary to specify the type of the distributions $\sigma_{I}$ and $P(K)$ of the compound nuclei over $I$ and $K$, respectively.

If one assumes that the quantity $\sigma_{I}$ is known, then the problem of angular distribution calculation consists only in the determination of the distribution $P(K)$. In the SPTS and SCTS models an equilibrium distribution of $K$ values is assumed which is determined by the Boltzmann factor $\exp \left(-E_{\text {rot }} / T\right)$ [3] at the saddle or scission point, respectively. Here, the rotational energy $E_{\text {rot }}$ is given by

$$
E_{\mathrm{rot}}(\mathbf{q}, I, K)=\frac{\hbar^{2} K^{2}}{2 J_{\|}}+\frac{\hbar^{2}\left(I(I+1)-K^{2}\right)}{2 J_{\perp}}=\frac{\hbar^{2} I(I+1)}{2 J_{\perp}}+\frac{\hbar^{2} K^{2}}{2 J_{\mathrm{eff}}} .
$$

Thus, the $K$ equilibrium distribution is

$$
P_{\text {eq }}(K)=\frac{\exp \left(-K^{2} / 2 K_{0}^{2}\right)}{\sum_{K=-I}^{I} \exp \left(-K^{2} / 2 K_{0}^{2}\right)} .
$$

The parameter $K_{0}$ determines the width of this distribution:

$$
K_{0}^{2}=\frac{T}{\hbar^{2}} J_{\text {eff }}, \quad J_{\text {eff }}=\frac{J_{\|} J_{\perp}}{J_{\perp}-J_{\|}},
$$

where the temperature $T$ and the effective moment of inertia $J_{\text {eff }}$ are those at the transition point.

By averaging (6) over $P_{\text {eq }}(K)$ from equation (9), one obtains an expression for the angular distribution with a fixed $I$ :

$$
W(\theta, I)=(I+1 / 2) \frac{\sum_{K=-I}^{K=I}\left|d_{0, K}^{I}(\theta)\right|^{2} \exp \left(-K^{2} / 2 K_{0}^{2}\right)}{\sum_{K=-I}^{K=I} \exp \left(-K^{2} / 2 K_{0}^{2}\right)} .
$$

At the same time, the variance of the $K$ values is given by

$$
\left(\sigma_{K}^{2}\right)_{\mathrm{eq}}=\sum_{K=-I}^{K=I} K^{2} P_{\mathrm{eq}}(K)=\frac{\sum_{K=-I}^{K=I} K^{2} \exp \left(-K^{2} / 2 K_{0}^{2}\right)}{\sum_{K=-I}^{K=I} \exp \left(-K^{2} / 2 K_{0}^{2}\right)} .
$$

Let us note that in the general case the variance of the $K$ distribution is not equal to $K_{0}^{2}$, but for the large values of $I$ one has $\left(\sigma_{K}^{2}\right)_{\mathrm{eq}} \simeq K_{0}^{2}$. 
As mentioned above, neither of the SPTS or SCTS model reproduces simultaneously well the angular distributions for the reactions with both heavy and light ions. The existent uncertainty with the position of the transition state indicates that it is necessary to take into account the dynamical features of the angular distribution formation. In the most general case, the tilting mode should be considered as an independent collective coordinate, and its evolution can be studied using, for example, the multidimensional Langevin approach [12]. Such a completely dynamical approach makes it possible to determine, in the most general form, the distribution $P(K)$. However, in this case, the problem of calculating the conservative force for the tilting mode, as well as the transport parameters (inertial, friction and diffusion parameters), arises. At the same time, a method for use in the calculation of the transport coefficients for the tilting mode has not been described in previous publications. Therefore, a fully dynamical consideration of the evolution of the $K$ degree of freedom is still difficult.

However, the dynamical aspects of the angular distribution formation can be contained within a characteristic named the tilting mode relaxation time $\tau_{K}$. In [32], it was proposed that the evolution of the tilting mode be considered using the Monte Carlo method. In [32], the fission process was characterized by two collective degrees of freedom: the elongation of the nucleus and $K$.

The dynamical model of the calculation of the fission fragment angular distributions proposed in $[32,33]$ was generalized here to the case of three-dimensional Langevin model. In the present model, the fission dynamics described within the Langevin approach by the three collective coordinates determining the shape of the compound nucleus $\left(c, h, \alpha^{\prime}\right)$ [9-12] was joined with the stochastic dynamics of the tilting mode. Thus, such a four-dimensional dynamical model of the fission process allows us to calculate the fission fragment angular distributions dynamically along with fission characteristics which can be calculated by the three-dimensional Langevin approach [9-12].

We use the following algorithm in order to describe the evolution of the tilting mode during the motion from the ground state to scission. The initial values of $K$ are chosen by the Monte Carlo procedure with equilibrium distribution (9) which for the spherical initial shape of a compound nucleus leads to a uniform distribution in the range $[-I, I]$. On each integration time step $\tau$ of the Langevin equations, we calculate the probability of changing of the $K$ value. For this, a uniformly distributed random number $\xi$ is sampled over the segment $[0,1]$. This number is compared with the ratio $\tau / \tau_{K}$. If $\xi<\tau / \tau_{K}$ then on the next time step a new value for $K=K^{\prime}$ is chosen. Otherwise, the previous value of $K$ is retained. A new configuration $K^{\prime}$ is chosen by importance sampling where the transition probability $w_{K K^{\prime}}$ is given by the Metropolis function [34]:

$$
w_{K K^{\prime}}= \begin{cases}\exp \left(-\Delta E_{K K^{\prime}} / T\right), & \Delta E_{K K^{\prime}}>0 \\ 1, & \Delta E_{K K^{\prime}} \leqslant 0\end{cases}
$$

where

$$
\Delta E_{K K^{\prime}}=E_{\text {rot }}\left(\mathbf{q}, I, K^{\prime}\right)-E_{\text {rot }}(\mathbf{q}, I, K) .
$$

One can find a more detailed description and a basis for the application of this method in review [35]. This Monte Carlo method is known as the Metropolis algorithm.

The relation of the Metropolis algorithm with stochastic kinetics can be explained on the basis of dynamical interpretation of this algorithm. It is known [36] that the algorithm describes the Markovian relaxation process characterized by the non-equilibrium distribution function $P_{K}(t) \equiv P(K, t)$ which satisfies the master equation:

$$
\frac{\mathrm{d} P_{K}(t)}{\mathrm{d} t}=-P_{K}(t) \sum_{K^{\prime}} w_{K K^{\prime}}+\sum_{K^{\prime}} w_{K^{\prime} K} P_{K^{\prime}}(t)
$$


where $w_{K K^{\prime}}$ is the probability of the transition of the system from the state with the projection $K$ to the state with the projection $K^{\prime}$. In order to the Markovian relaxation process described by equation (14) has the necessary property of convergence to the equilibrium state of the canonical ensemble the detailed balance condition must be satisfied: $w_{K K^{\prime}} P_{\text {eq }}(K)=$ $w_{K^{\prime} K} P_{\mathrm{eq}}\left(K^{\prime}\right)$. This relation does not determine the function $w_{K K^{\prime}}$ unambiguously. The function $w_{K K^{\prime}}$ is usually chosen in the form of the Metropolis function given by equation (13) or by the Glauber function [37]:

$$
w_{K K^{\prime}}=\frac{1-\operatorname{th}\left(\Delta E_{K K^{\prime}} / 2 T\right)}{2}
$$

All the present calculations were carried out with the Metropolis function (13). The relation $\left\langle A_{K}(t)\right\rangle=\sum_{K} A_{K} P_{K}(t)$ determines the dynamical evolution of the quantity $A_{K}$ by means of the function $P_{K}(t)$ - the solution of equation (14).

Under certain conditions and approximations (see details in [38]), master equation (14) can be transformed first to the Fokker-Planck equation and then to the reduced Langevin equation which describes the relaxation diffusion process of overdamped motion. This is understandable since master equation (14) contains no inertial terms [38].

In the present work, the values of $I$ and $K$ were recorded both at the saddle and scission configurations for each Langevin dynamical trajectory which reached the scission configuration. So, one could analyse the $K$ distributions for the ensemble of fission trajectories at the distinctive points of the potential energy (at the saddle and scission points). One can calculate the angular distribution of fission fragments by averaging equation (6) over the ensemble of Langevin trajectories at scission configuration:

$$
\langle W(\theta)\rangle=\frac{1}{N_{\mathrm{f}}} \sum_{j=1}^{N_{\mathrm{f}}}\left(I^{j}+1 / 2\right)\left|d_{0 K^{j}}^{I^{j}}(\theta)\right|^{2},
$$

where $I^{j}, K^{j}$ are the angular momentum for the $j$-trajectory and its projection on the fission axis, which determine the angular distribution formation. $N_{\mathrm{f}}$ is the number of fission events, $\theta$ is the angle between the fission axis and the beam axis.

In the present paper, equation (16) was used to calculate the angular distribution of fission fragments and, in particular, the anisotropy of the angular distribution that is defined by the ratio $\left\langle W\left(0^{\circ}\right)\right\rangle /\left\langle W\left(90^{\circ}\right)\right\rangle$.

The relaxation time of the tilting mode $\tau_{K}$ was taken as a free parameter in our approach. The parameter $\tau_{K}$ was assumed to be constant during the evolution of the nucleus from the ground state to scission configuration. However, in general, the relaxation time $\tau_{K}$ is not a constant. It was pointed out in [39] that $\tau_{K}$ depends on the effective moment of inertia and, consequently, on the shape of the fissioning nucleus. But the explicit form for this dependence is still unknown, and it could not be found within the present model.

It is necessary to note that this approach based on the Metropolis algorithm is general in the sense that it allows one to consider the evolution of an arbitrary finite collective mode of nuclear fission in a similar way. For example, one can similarly use it to describe the evolution of mass-asymmetry and charge-asymmetry modes. The application of the dynamical approach based on the Metropolis algorithm to the modes mentioned above is of special interest for studies due to the well-studied dependences of their relaxation times on the elongation parameter [40]. 


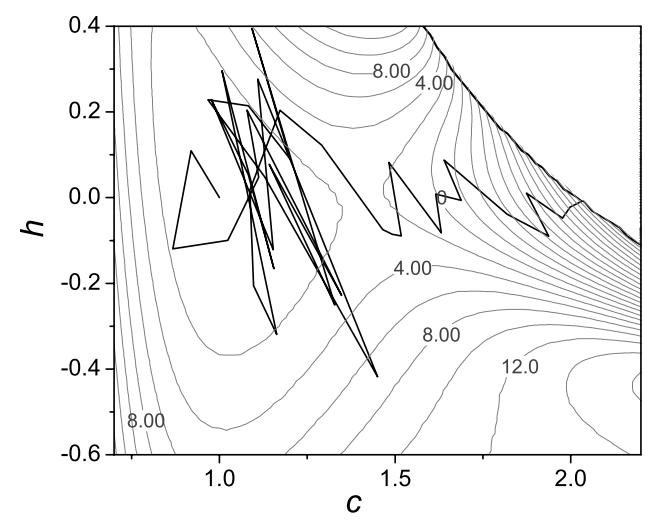

Figure 1. Stochastic Langevin trajectory in the space of the collective coordinates $\left(c, h, \alpha^{\prime}=0\right)$ is shown against the potential-energy background. The numbers at the isolines specify the values of the potential energy in MeV. The solid line in the right upper corner of the figure is the scission line. The trajectory given in this figure represents a fission event.

\section{Results of calculations and discussion}

\subsection{Test of dynamical approach to the treatment of angular distribution of fission fragments in equilibrium conditions}

An example of a Langevin trajectory is shown in figure 1. The initial deformation of nucleus is chosen as spherical ( $c=1 ; h=0$ ) and numerical integration of the Langevin equations is finished when the system reaches the scission configuration. Calculations of the observables are carried out by modelling an ensemble of Langevin trajectories and further by statistical averaging over the ensemble. An illustration is presented for the ${ }^{248} \mathrm{CF}$ nucleus formed in the reaction ${ }^{16} \mathrm{O}+{ }^{232} \mathrm{Th}$ with zero angular momentum. The potential energy surface has been calculated within the finite-range drop model with parameters taken from [20].

Figure 2 shows the evolution of the tilting mode $K$ during the motion of the compound nucleus from the ground state to scission configuration. As mentioned above, the initial $K$ distribution is taken as a uniform distribution on the interval $[-I, I]$. It is seen from the figure that the number of the changing of the tilting spin depends substantially on the relaxation time $\tau_{K}$. The $K$ distribution keeps 'frozen' from the initial ground state deformation till scission for the large values of $\tau_{K}$ (in comparison with the integration time step $\tau$ of the Langevin equations). At the same time, the value of $K$ changes practically on each time step $\tau$ for $\tau_{K} \ll \tau$. It means that the system has no memory about the $K$ distribution in the case $\tau_{K} \ll \tau$ (i.e., the system forgets the former $K$ distribution).

We have examined the following limiting assumption in order to test the present dynamical approach. We fixed the value of the relaxation time of the tilting mode $\tau_{K}$ to be shorter than the integration time step of the Langevin equations $\tau$. In this case, $\tau_{K}$ is also much shorter than the characteristic times of the fission process:

$$
\tau_{K}<\tau, \quad \tau_{K} \ll \tau_{\mathrm{gs}-\mathrm{sd}}, \quad \tau_{K} \ll \tau_{\mathrm{sd}-\mathrm{sc}},
$$

where $\tau_{\mathrm{gs}-\mathrm{sd}}, \tau_{\mathrm{sd}-\mathrm{sc}}$ are the mean time of the evolution of the compound nucleus from its ground state to the saddle configuration and the mean time of the descent from the saddle to scission configuration, correspondingly. Involving this assumption one can expect that the 


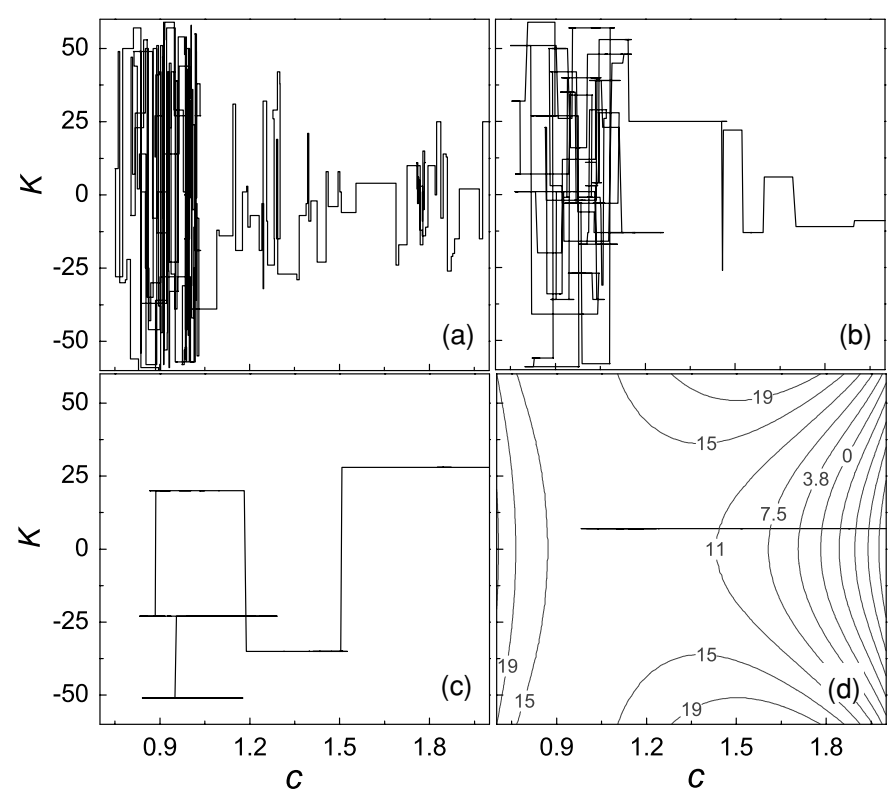

Figure 2. Examples of evolution trajectories of the tilting mode $K$ during the motion of the compound nucleus ${ }^{248} \mathrm{Cf}$ with total angular momentum $I=60 \hbar$ from the ground state to scission configuration along the elongation coordinate $c$ for the different values of $\tau_{K}$. The values of the relaxation time of the tilting mode $\tau_{K}$ in this figure are: $0.1 \times 10^{-21} \mathrm{~s}(\mathrm{a}), 1 \times 10^{-21} \mathrm{~s}(\mathrm{~b}), 10 \times 10^{-21}$ $\mathrm{s}(\mathrm{c})$ and $100 \times 10^{-21} \mathrm{~s}(\mathrm{~d})$. All the trajectories have the same potential-energy background as shown in (d). The numbers at the isolines specify the values of the potential energy in MeV.

tilting mode reaches a thermodynamical equilibrium with the thermostat on each integration time step of the Langevin equations.

Under this limiting assumption, one should expect that the variance of the dynamical $K$ distribution obtained within the present model at the saddle and scission configurations by the equation

$$
\left(\sigma_{K}^{2}\right)_{\mathrm{dyn}}=\left\langle K^{2}\right\rangle-\langle K\rangle^{2}
$$

will be close to the equilibrium values $\left(\sigma_{K}^{2}\right)_{\text {eq }}$, predicted by the SPTS and SCTS theories (equation (12)), correspondingly. Therefore, one should also expect that the angular anisotropies $\left\langle W\left(0^{\circ}\right)\right\rangle /\left\langle W\left(90^{\circ}\right)\right\rangle$ defined by equation (16) at the saddle and scission configurations will be close to the results calculated by equation (11) from the SPTS and SCTS models, correspondingly.

The results for the reaction ${ }^{16} \mathrm{O}+{ }^{238} \mathrm{U}, E_{\mathrm{lab}}=148 \mathrm{MeV}$, obtained with $\tau_{K}=0.001 \times$ $10^{-21} \mathrm{~s}$, are shown in figure 3. The prediction of the SPTS and SCTS models are also shown in this figure. It can be seen from this figure that the variance of the $K$ distribution calculated by both the dynamical (at the saddle and scission configurations) and the statistical (SPTS and SCTS) models is in close agreement. At the same time, the anisotropies of the fission fragment angular distribution obtained dynamically and the predictions of the statistical models are different on $0.5-1.0 \%$.

Thus, the obtained results show that the developed model is accurate enough for the calculation of the fission fragment angular distribution. However, it should be noted that $(50-60) \times 10^{3}$ trajectories are minimum statistics that is necessary in order to reach the 

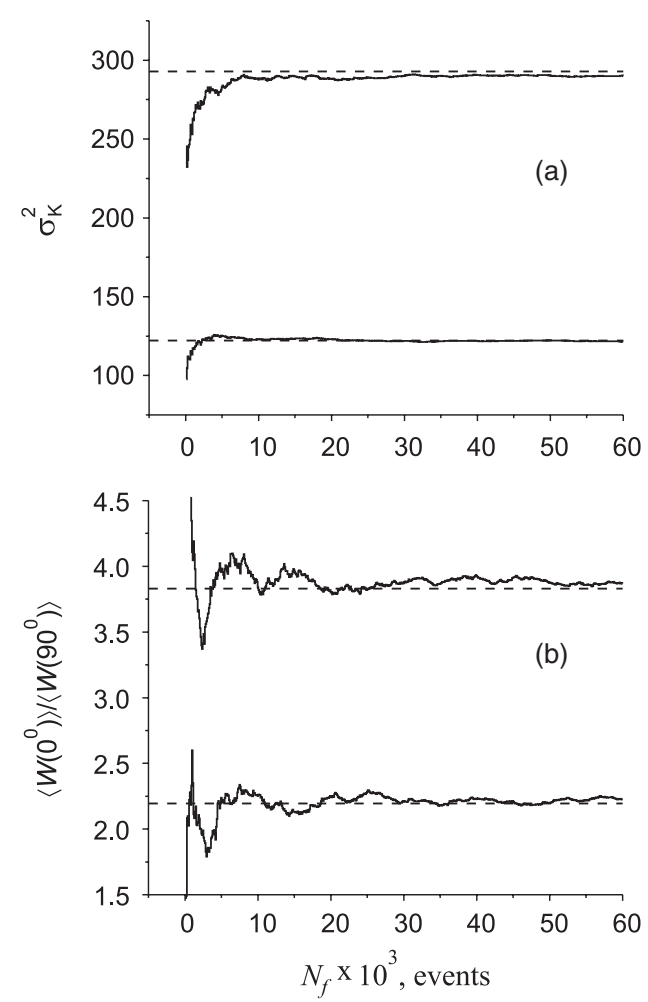

Figure 3. The variance of the dynamical $K$ distribution (a) and the anisotropy of the angular distribution (b), calculated at the saddle (the upper curve in (a) and lower curve in (b)) and scission (the lower curve in (a) and upper curve in (b)) configurations as functions of the number of fission events simulated in calculations. The dashed curves represent the statistical limits obtained within the SPTS and SCTS models.

convergence of the solution for the anisotropy of the angular distribution. This can be explained by the fact that only the trajectories with $K=0$ give a nonzero contribution to the anisotropy of fission fragments. However, these trajectories are few among all the trajectories.

\subsection{Comparison with experimental data}

The results of the analysis of the experimental data on the fission fragment angular distribution have been obtained for the reactions with oxygen as a projectile and for the energies above the Coulomb fusion barrier ${ }^{16} \mathrm{O}+{ }^{232} \mathrm{Th} \rightarrow{ }^{248} \mathrm{Cf},{ }^{16} \mathrm{O}+{ }^{238} \mathrm{U} \rightarrow{ }^{254} \mathrm{Fm},{ }^{16} \mathrm{O}+{ }^{248} \mathrm{Cm} \rightarrow{ }^{264} \mathrm{Rf}$. Such a choice of a projectile is motivated by the fact that for the reactions with a projectile with $A<20$ the dominating channel is the fusion-fission one while the quasifission processes are suppressed appreciably. On the other hand, for these reactions a substantial part of the initial distribution of the compound nucleus on the angular momenta leads to fission barriers comparable or even less than the nucleus temperature. The applicability of the SPTS model is doubtful in this case, and, therefore, the analysis of the experimental data for the chosen reactions is a good opportunity for the application of the developed approach.

We have analysed the experimental data on the angular distributions and energy dependences of the anisotropy for the mentioned three reactions. The results of calculations 


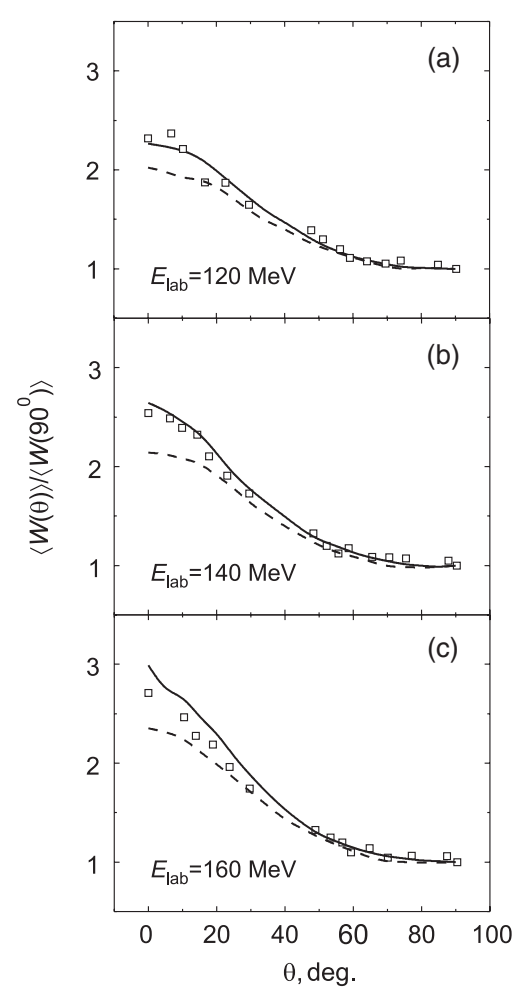

Figure 4. Comparison of experimental data on the angular distributions of fission fragments (open squares) with the results of present model calculations for the reaction ${ }^{16} \mathrm{O}+{ }^{232} \mathrm{Th} \rightarrow{ }^{248} \mathrm{Cf}$ at various projectile laboratory energies. The experimental data are taken from [27]. The curves represent theoretical calculations with $\tau_{K}=4 \times 10^{-21} \mathrm{~s}$ (solid curves) and $\tau_{K}=8 \times 10^{-21} \mathrm{~s}$ (dashed curve)

of the angular distributions are shown in figures 4 and 5. The angular distributions calculated with $\tau_{K}=4 \times 10^{-21} \mathrm{~s}$ and $\tau_{K}=8 \times 10^{-21} \mathrm{~s}$ for the reaction ${ }^{16} \mathrm{O}+{ }^{232} \mathrm{Th} \rightarrow{ }^{248} \mathrm{Cf}$ are shown in figure 4 . It can be seen from figure 4 that the angular distribution is sensitive to the value of the relaxation time of the tilting mode.

Figure 6 shows the anisotropy of the fission fragment angular distribution calculated with $\tau_{K}=4 \times 10^{-21} \mathrm{~s}$ as a function of the projectile laboratory energy $E_{\text {lab. One can see }}$ from figures 4-6 that the present calculations of the angular distributions and anisotropies of the fission fragment angular distributions performed with $\tau_{K}=4 \times 10^{-21} \mathrm{~s}$ agree well with the experimental data for a number of compound nuclei in a wide range of projectile energy. It is clearly seen from the figures that the dynamical calculations give results closer to the predictions of the SPTS model than to the predictions of the SCTS model. It can be interpreted as a consequence of the relation of the characteristic times: $\tau_{\mathrm{gs}-\mathrm{sd}}$ and $\tau_{\mathrm{sd}-\mathrm{sc}}$. For the value of the reduction coefficient $k_{s}$ that have been used in this study and for the considered heavy fissioning nuclei, we have the following values of the characteristic times: $\tau_{\mathrm{gs}-\mathrm{sd}} \sim(9-15) \times 10^{-21} \mathrm{~s}$ and $\tau_{\mathrm{sd}-\mathrm{sc}} \sim 6 \times 10^{-21} \mathrm{~s}$. The relaxation time of the tilting mode $\tau_{K}$ is not much shorter than these values. Therefore, one could not expect that the equilibrium distribution of the tilting mode is reached at scission configuration. On the other hand, the used value of $\tau_{K}$ is comparable with both $\tau_{\mathrm{gs}-\mathrm{sd}}$ and $\tau_{\mathrm{sd}-\mathrm{sc}}$. Therefore, remembering of the former 


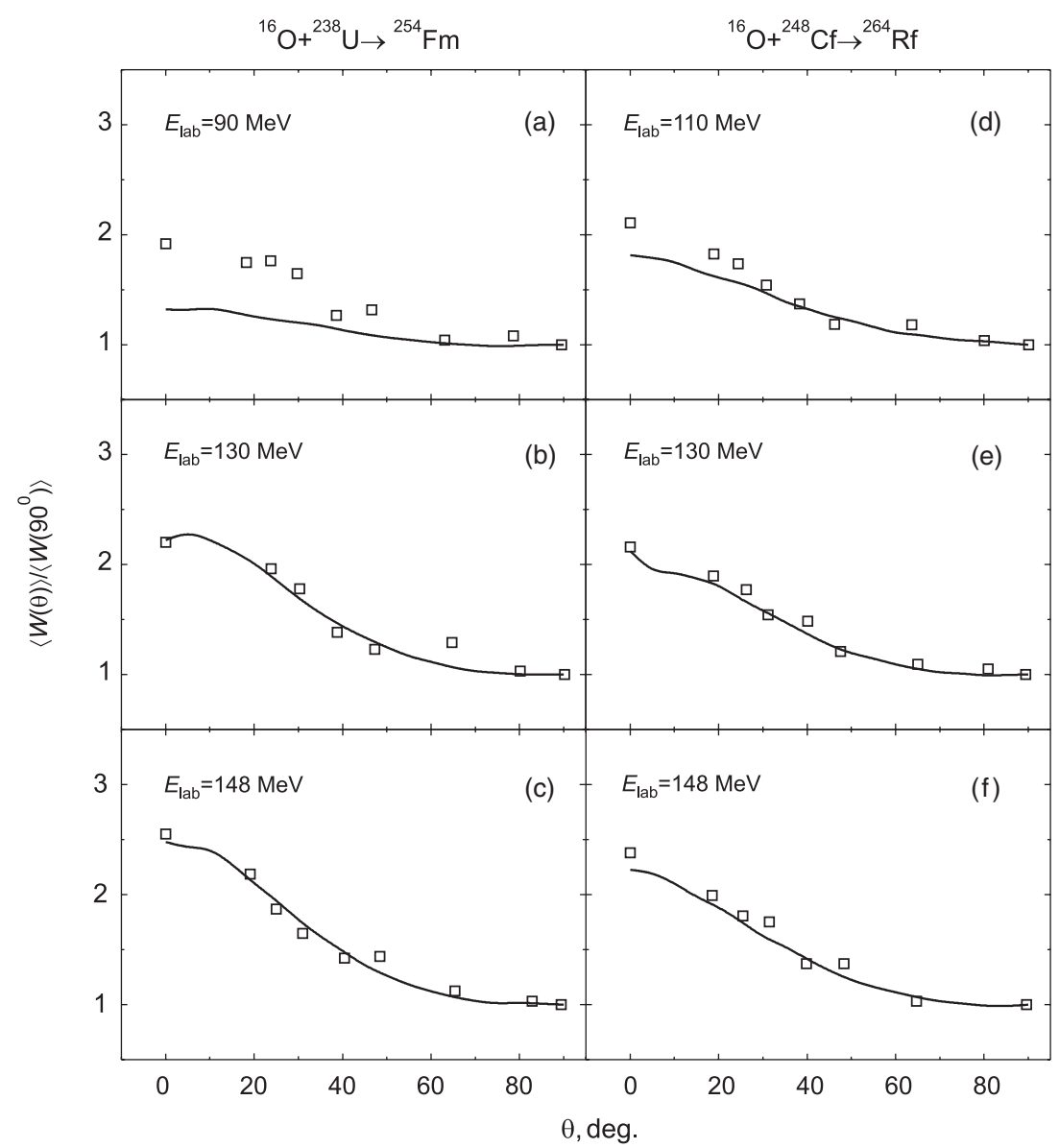

Figure 5. Comparison of experimental data on the angular distribution of fission fragments (open squares) with the results of present model calculations for the reactions: ${ }^{16} \mathrm{O}+{ }^{238} \mathrm{U} \rightarrow{ }^{254} \mathrm{Fm}$ (a) (c) and ${ }^{16} \mathrm{O}+{ }^{248} \mathrm{Cm} \rightarrow{ }^{264} \mathrm{Rf}(\mathrm{d})-(\mathrm{f})$ at various projectile laboratory energies. The experimental data are taken from [27]. The solid curves represent theoretical calculations with $\tau_{K}=4 \times$ $10^{-21} \mathrm{~s}$.

distribution of the tilting mode at the saddle point and even at the deformations before the saddle point can take place and determines the resulting fission fragment angular distribution at scission.

It should be noted that the earlier obtained estimates of $\tau_{K}$ lie in a rather wide interval $(5-8) \times 10^{-21} \mathrm{~s}[33,41-43]$ to $60 \times 10^{-21} \mathrm{~s}$ [44]. We stress that the relaxation time $\tau_{K}$ was determined in [33] within a one-dimensional Langevin model, while, in other studies, calculations were performed within statistical models. At the same time, it follows from [39] that there is a dependence of $\tau_{K}$ on the velocity of nuclear rotation, i.e. considering the tilting mode relaxation time as a constant is only an approximation. Until now, there have been no dynamical calculations performed using this result. The results of [39] were applied to the analysis of the angular distribution in the framework of the statistical model proposed in [45]. The authors of these studies achieved reasonably good agreement with the experimental data in the region of near-barrier and sub-barrier energies in the entrance channel of the reaction of heavy-ion collisions. 

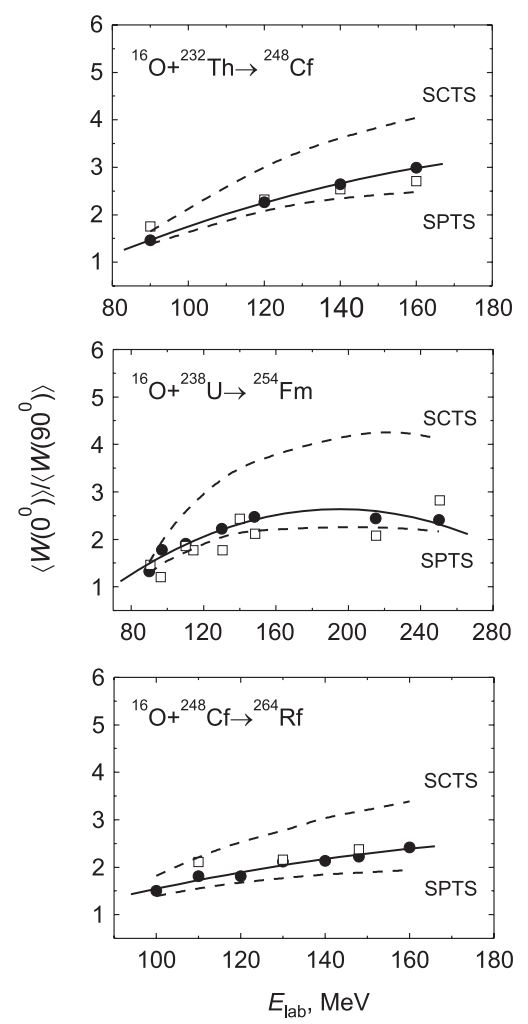

Figure 6. The anisotropy of the fission fragment angular distribution as a function of projectile laboratory energy. The open squares are the experimental data from [27-31]. The filled circles represent the theoretical calculations with $\tau_{K}=4 \times 10^{-21} \mathrm{~s}$. The solid curves are an approximation of theoretical calculations by a polynomial of second order. The dashed lines give the theoretical predictions of the SPTS and SCTS models.

\section{Summary and conclusions}

Three-dimensional Langevin dynamics was joined with Monte Carlo algorithm of the calculation of the fission fragment angular distribution that takes into account the stochastic nature of the fission process and the formation of the angular distribution. The energy dependence of the anisotropy of the fission fragment angular distribution revealed from the present model that calculations were found to be closer to the experimental data than the predictions of the statistical models SPTS and SCTS. In the present approach, the relaxation time of the tilting mode was estimated. The anisotropies of the fission fragment angular distributions obtained with $\tau_{K}=4 \times 10^{-21} \mathrm{~s}$ agree well with the experimental data. Such a phenomenologically estimated value for $\tau_{K}$ is comparable with the mean time of the descent from the saddle point to scission $\left(\tau_{\mathrm{sd}-\mathrm{sc}} \sim 6 \times 10^{-21} \mathrm{~s}\right)$. This fact points out that the $K$ distribution changes during the descent from the saddle to scission, and, on the other hand, that the classical statistical theory of the transition state is inapplicable in this situation.

In summary, it would be desirable to emphasize that the elucidation of the role of the dynamical factors influenced on the formation of the angular distribution is on the initial stage only. The present dynamical approach allowed us to obtain reasonable agreement of the results 
of calculations with experimental angular distributions. The use of the multidimensional Langevin model joined with the Monte Carlo algorithm, which considers the tilting mode as an independent collective degree of freedom, seems to be promising.

\section{Acknowledgments}

We would like to thank Dr P N Nadtochy for the valuable comments, suggestions and careful reading of the manuscript. We are grateful to Dr K-H Schmidt for useful and interesting discussions.

\section{References}

[1] Vandenbosch R and Huizenga J R 1973 Nuclear Fission (New York: Academic) p 422

[2] Bohr A 1956 Proc. United Nations International Conference on the Peaceful Uses of Atomic Energy (New York), vol 2 p 151

[3] Halpern I and Strutinsky V M 1958 Proc. Second United Nations International Conference on the Peaceful Uses of Atomic Energy (Geneva), vol 15 p 408

[4] Bond P D 1984 Phys. Rev. Lett. 52414

Bond P D 1985 Phys. Rev. C 32471

Bond P D 1985 Phys. Rev. C 32483

[5] Rossner H H, Huizenga J R and Schröder W U 1984 Phys. Rev. Lett. 5338

Rossner H H, Huizenga J R and Schröder W U 1986 Phys. Rev. 33560

[6] John B and Kataria S K 1998 Phys. Rev. 571337

[7] Vaz L C and Alexander J M 1983 Phys. Rep. 971

[8] Freifelder R, Prakash M and Alexander J M 1986 Phys. Rep. 133315

[9] Karpov A V, Nadtochy P N, Vanin D V and Adeev G D 2001 Phys. Rev. C 63054610

[10] Nadtochy P N, Adeev G D and Karpov A V 2002 Phys. Rev. C 65064615

[11] Nadtochy P N, Karpov A V, Vanin D V and Adeev G D 2003 Yad. Fiz. 661240

[12] Adeev G D, Karpov A V, Nadtochy P N and Vanin D V 2005 Fiz. Elem. Chastits At. Yadra 36732

[13] Ryabov E G, Karpov A V and Adeev G D 2006 Nucl. Phys. A 76539

[14] Brack M, Damgaard J, Jensen A S, Pauli H C, Strutinsky V M and Wong CY 1972 Rev. Mod. Phys. 44320

[15] Fröbrich P and Gontchar I 1998 Phys. Rep. 292131

[16] Ignatyuk A V, Itkis M G, Okolovich V N, Smirenkin G N and Tishin A S 1975 Yad. Fiz. 211185

[17] Karpov A V, Ryabov E G, Nadtochy P N and Adeev G D 2003 J. Phys. G: Nucl. Part. Phys. 292365

[18] Krappe H J 1999 Phys. Rev. C 592640

[19] Krappe H J, Nix J R and Sierk A J 1979 Phys. Rev. C 20992

[20] Sierk A J 1986 Phys. Rev. C 332039

[21] Davies K T R and Nix J R 1976 Phys. Rev. C 141977

[22] Nix J R and Sierk A J 1987 Proc. Int. School-Seminar on Heavy-Ion Physics (Dubna, USSR) p 453

[23] Nix J R and Sierk A J 1987 Proc. 6th Adriatic Conference on Nuclear Physics: Frontiers of Heavy Ion Physics (Dubrovnik, Yugoslavia) p 333

[24] Marten J and Fröbrich P 1992 Nucl. Phys. A 545854

[25] Davies K T R, Managan R A, Nix J R and Sierk A J 1977 Phys. Rev. C 161890

[26] Mavlitov N D, Fröbrich P and Gontchar I I 1992 Z. Phys. A 342195

[27] Back B B, Betts R R, Gindler J E, Wilkins B D, Saini S, Tsang M B, Gelbke C K, Lynch W G, McMahan M A and Baisden P A 1985 Phys. Rev. C 32195

[28] Vaz L C, Logan D, Duek E, Alexander J M, Rivet M F, Zisman M S, Kaplan M and Ball J W 1984 Z. Phys. A 315169

[29] Gavron A, Eskola P, Sierk A J, Boissevain J, Britt H C, Eskola K, Fowler M M, Ohm H and Wilhelmy J B 1984 Phys. Rev. Lett. 52589

[30] Karamyan S A, Kuznetsov I V, Muzycka Yu A, Oganessian Yu Ts, Penionzkevich Yu E and Pustyl'nik B I 1967 Yad. Fiz. 6494

[31] Töke J et al 1984 Phys. Lett. B 142258

[32] Drozdov V A, Eremenko D O, Fotina O V, Giardina G, Malaguti F, Platonov S Yu and Yuminov O A 2004 Nucl. Phys. A 734225 
Drozdov V A, Eremenko D O, Fotina O V, Platonov S Yu and Yuminov O A 2003 Book of Abstracts of VII International Conference on Nucleus-Nucleus Collisions (Moscow, Russia, 17-21 June) (Dubna: JINR) p 171

[33] Drozdov V A, Eremenko D O, Platonov S Yu, Fotina O V and Yuminov O A 2001 Yad. Fiz. 64221

[34] Metropolis N, Rosenbluth A, Rosenbluth M, Teller A and Teller E 1953 J. Chem. Phys. 211087

[35] Binder K 1997 Rep. Prog. Phys. 60487

[36] Binder K and Heerman W 1988 Monte-Carlo Simulation in Statistical Physics (Berlin: Springer)

[37] Glauber R J 1963 J. Math. Phys. 4294

[38] Weindenmuller H A 1980 Progr. Part. Nucl. Phys. 349

[39] Døssing T and Randrup J 1985 Nucl. Phys. A 433215

[40] Adeev G D, Gonchar I I, Pashkevich V V and Serdyuk O I 1989 Yad. Fiz. 501242

Karpov A V and Adeev G D 2002 Eur. J. Phys. A 14169

[41] Ramamurthy V S and Kapoor S S 1985 Phys. Rev. Lett. 54178

[42] Butler M A, Datta S S, de Souza R T, Huizenga J R, Schröder W A, Töke J and Wile J L 1986 Phys. Rev. C 342016

[43] Newton J O 1990 Fiz. Elem. Chastits At. Yadra 21821

Oganessian Yu Ts and Lazarev Yu A 1985 Treatise on Heavy Ion Science vol 4, ed D A Bromley (New York: Plenum) p 1

[44] Thomas R G, Choudhury R K, Mohanty A K, Saxena A and Kapoor S S 2003 Phys. Rev. C 67041601

[45] Liu Z, Zhang H, Xu J, Xu J, Qiao Yu, Qian X and Lin C 1995 Phys. Lett. B 353173

Zhang H Q, Liu Z H, Xu J C, Ruan M, Lin C J and Qian X 2002 J. Nucl. Radiochem. Sci. 399 